\title{
Article \\ Effects of Several Chemicals on the Migration Behavior of Bursaphelenchus xylophilus (Steiner \& Buhrer) Nickle
}

\author{
Xiu-Feng Shi ${ }^{1,2}$, Xiao-Lei Ding ${ }^{1,2}$, Li-Na Shi ${ }^{1,2}$, Qing-Tong Wang ${ }^{1,2}$ and Jian-Ren Ye ${ }^{1,2, *}$ \\ 1 Co-Innovation Center for Sustainable Forestry in Southern China, College of Forestry, Nanjing Forestry \\ University, Nanjing 210037, China; xiufengshi@njfu.edu.cn (X.-F.S.); dxl@njfu.edu.cn (X.-L.D.); \\ sln@njfu.edu.cn (L.-N.S.); qtwang@njfu.edu.cn (Q.-T.W.) \\ 2 Jiangsu Key Laboratory for Prevention and Management of Invasive Species, Nanjing 210037, China \\ * Correspondence: jrye@njfu.edu.cn; Tel.: +86-139-0515-1921; Fax: +86-25-8542-7305
}

check for

updates

Citation: Shi, X.-F.; Ding, X.-L.; Shi, L.-N.; Wang, Q.-T.; Ye, J.-R. Effects of Several Chemicals on the Migration Behavior of Bursaphelenchus xylophilus (Steiner \& Buhrer) Nickle. Forests 2021, 12, 771. https://doi.org/ 10.3390/f12060771

Received: 12 April 2021

Accepted: 8 June 2021

Published: 11 June 2021

Publisher's Note: MDPI stays neutral with regard to jurisdictional claims in published maps and institutional affiliations.

Copyright: (c) 2021 by the authors. Licensee MDPI, Basel, Switzerland. This article is an open access article distributed under the terms and conditions of the Creative Commons Attribution (CC BY) license (https:// creativecommons.org/licenses/by/ $4.0 /)$.

\begin{abstract}
The pine wood nematode (PWN), Bursaphelenchus xylophilus, that causes pine wilt disease (PWD), has severely damaged forests and ecosystems in countries of East Asia and Europe. Since the high propagation rate of PWD is the main cause of the devastating disease, it is very important to interfere with the spread of PWN mediated by its insect vector (Monochamus alternatus). In order to explore new methods and approaches for the prevention and control of pine wood nematode disease, chemicals with attraction or repellency to pine wood nematode and stable diffusion or volatilization in infected wood were screened out to interfere and hinder the pupa chamber effect, the aggregation behavior of PWN to pupa chamber in M. alternatus. In the present study, 22 chemicals were inoculated on water agar medium and infected wood to verify the tropism of PWN toward the chemicals from the aspects of attraction and repellency. The results indicate that linoleic acid, oleic acid, diacetyl and $\beta$-myrcene have attractive effects on PWN, while kerosene and $\beta$-humulene have repellent effects on PWN and are able to stably permeate and volatilize within pine trees. This research shows that some chemicals can affect the movement behavior of PWN and interfere with its migration to the pupal chamber in M. alternatus, thus reducing the dispersal efficiency of PWNs carried by insect vectors. This chemically based strategy would provide novel approaches for effective controlling of pine wilt disease.
\end{abstract}

Keywords: pine wood nematode; insect vector; repellent effect; attractive effect

\section{Introduction}

Pine wood nematode (PWN) Bursaphelenchus xylophilus (Steiner and Buhrer, 1934) Nickle, 1970. is known to originate in North America and first spread to Japan in the early 1900s and dispersed to other parts of Asia (China, Japan, and South Korea) and Europe (including Portugal and Spain) [1]. The infected pine trees by PWN leads to blockage of the tree vasculature, resulted in wilting symptoms and ultimately death [2]. A susceptible host can wilt and die within weeks of infection if conditions are favorable for disease development. Despite the tremendous efforts to control pine wilt disease (PWD) and quarantine wood products from infested areas, PWD has become the most destructive threat to all forests in Asia.

The main reason that PWD has such a great impact on the ecological environment is its high infectivity [3]. The dispersal ways of PWN can be divided into natural dispersal and human-mediated dispersal [4]. Natural dispersal depends mainly on the insect vectors which considered as the most common ways to transmit the nematode [1]. (Monochamus spp.) Each Monochamus alternatus Hope individual is able to carry thousands to tens of thousands of PWNs. The life cycle of PWN includes two stages: the reproduction stage and the dispersal stage [5]. The dispersal of the PWN occurs when the PWN develops to the fourth larval (dispersal) stage $\left(\mathrm{J}_{\mathrm{IV}}\right)$ upon encountering its insect vector, the pine sawyer beetle [6], inside an infected pine tree. The life cycles of Bursaphelenchus xylophilus and M. 
alternatus are specifically coordinated with each other [7]. When conditions are unfavorable, such as when food resources and temperatures are low, individuals in the second larval stage $\left(\mathrm{J}_{\mathrm{II}}\right)$ molt to the third larval stage $\left(\mathrm{J}_{\mathrm{III}}\right)$ and aggregate around the pupal chamber of M. alternatus $[8,9]$. Before the emergence of adult longicorn beetles, $\mathrm{J}_{\mathrm{III}}$ larvae become the fourth-instar larvae, reaching the dispersal stage (JIV). Then, PWN enters the respiratory system of the adult longicorn beetles through the spiracles; at this time, the beetles are newly winged, and their body wall has not completely sclerotized [10]. Subsequently, when adult longicorn beetles take in supplementary nutrition or lay eggs, the PWNs escape from the trachea of longicorn beetles and enter the new host's body via wounds caused by the feeding or spawning of longicorn beetles.

Therefore, the coordinated life cycle between PWN and its insect vector as well as the accurate identification of the pupa chamber of longicorn were heated discussed by scholars. Chemical signals were proved to be a key role in mediating interspecific interactions among PWN, its vector beetle and the pine tree host. The aggregation of $\mathrm{L}_{\mathrm{III}}$ larvae around the beetle pupal chambers is triggered by terpene signals produced by the beetles when developing into adults [11]. The $\mathrm{L}_{\mathrm{III}}-\mathrm{L}_{\mathrm{IV}}$ molt in PWN is stimulated by fatty acid ethyl esters produced by beetles, specifically during the late pupa and early adult stages [12]. Furthermore, chemical signals also play an important role in mediating intraspecific interactions within populations of nematodes [12]. Chemicals that are highly attractive to PWNs, such as oleic acid and linoleic acid, were found in pine trees, beetle pupal chambers, larvae and adults of longicorns [13]. Many species of free-living and parasitic nematodes coordinate their behavior and development by secreting a family of pheromones called the ascaroside. The ascarosides have been shown to affect dauer formation, sexual attraction, aggregation, dispersal and olfactory plasticity in nematodes [14].

In this study, chemicals that not only have attractive or repellent effects on PWN but can also stably permeate and spread within infected wood were screened. The prospects of the study were to develop new strategies for blocking the infection cycle of PWN and to provide new ideas and methods for the prevention and control of PWD.

\section{Materials and Methods}

\subsection{Source and Culture of PWNs}

PWN isolates (AmA3, AA3, and YW4) were stored in the Forest Pathology Laboratory of Nanjing Forestry University. They were cultured on Botrytis cinerea in Potato Dextrose Agar (PDA) Medium at $25^{\circ} \mathrm{C}$ in the dark.

AmA3 was separated from susceptible Pinus thunbergii Parl. in Anhui Province, China; AA3 was separated from susceptible Pinus taiwanensis Hayata in Anhui Province, China; YW4 was separated from susceptible Pinus kesiya var. langbianensis in Yunnan Province, China [11].

\subsection{Chemicals}

Twelve chemicals with potential attraction effect and eleven chemicals with repellency effects are used in this study (Table 1).

The long-chain fatty acids were proved to be related to the migration of PWNs to the body and pupal chamber of its insect vector (M. alternatus) [7]. Diacetyl has a behavioral stimulus effect on Caenorhabditis elegans [12]. These chemicals and their homologues are used in this study.

\subsection{The Self-Designed Assay for Evaluating Attractive Effects of Chemicals toward PWN}

Water agar medium $[13,14]$ ( $15 \mathrm{~cm}$ diameter) was used to test the attractive effects of several chemicals toward PWNs (Figure 1). First, a hole was made on $3 \mathrm{~cm}$ from the edge of the plate, a sterile cotton ball was placed in the hole, and chemicals were dripped on the cotton ball. Another sterile cotton ball was placed in another hole at the corresponding position on the opposite side of the same dish, and a $200 \mu \mathrm{L}$ aliquot of worm solution 
(approximately 1000 PWNs) was added to the cotton ball. Sterile water treatment was used as the control.

Table 1. Purity and sources of the chemicals used for chemical identification and laboratory and field assays.

\begin{tabular}{|c|c|c|c|}
\hline Compound & Purity (\%) & CAS No. & Source \\
\hline $\mathrm{NaClO}$ & $5.2 \%$ & $7681-52-9$ & Damas-beta. \\
\hline $\mathrm{C}_{2} \mathrm{HCL}_{3} \mathrm{O}_{2}$ & $>99 \%$ & $200-663-8$ & Damas-beta \\
\hline Clycerol & $>99 \%$ & $56-81-5$ & Damas-beta \\
\hline Lactic acid & $\geq 97.5 \%$ & $50-21-5$ & General-reagent \\
\hline Palmitic acid & $98 \%$ & $57-10-3$ & General-reagent \\
\hline Stearic acid & $40 \%$ & $57-11-4$ & General-reagent \\
\hline$\beta$-Caryophyllene & $30 \%$ & $123-35-3$ & General-reagent \\
\hline$\beta$-Myrcene & $\geq 75 \%$ & $123-35-3$ & Mackin \\
\hline Toluene & $\geq 99.5 \%$ & $108-88-3$ & Mackin \\
\hline Oleic acid & $90 \%$ & $112-80-1$ & Mackin \\
\hline Linoleic acid & $\geq 98 \%$ & $60-33-3$ & Mackin \\
\hline Diacetyl & $\geq 98.0 \%$ & $431-03-8$ & Mackin \\
\hline $\mathrm{KCl}$ & $\geq 99.8 \%$ & $7447-40-7$ & Nanjing Chemical Reagent Co., Ltd. \\
\hline $\mathrm{NaC} 1$ & $\geq 99.5 \%$ & $7647-14-5$ & Nanjing Chemical Reagent Co., Ltd. \\
\hline $\mathrm{MnCl}_{2}$ & $98 \%$ & $7773-01-5$ & Nantong agro-chemical co. LTD \\
\hline $\mathrm{CrCl}_{2} \cdot 2.5 \mathrm{H}_{2} \mathrm{O}$ & $\geq 99.0 \%$ & $7790-78-5$ & Ourchen \\
\hline $\mathrm{NaF}$ & $\geq 99.0 \%$ & $7681-49-4$ & Sinopharm Chemical Reagent Co., Ltd. \\
\hline Atropine sulfate & $100 \mathrm{~m} \mu \mathrm{g} / \mathrm{mL}$ & $5908-99-6$ & Sinopharm Chemical Reagent Co., Ltd. \\
\hline DDVP & $200 \mathrm{~g} / \mathrm{L}$ & $203645-53-8$ & Sinopharm Chemical Reagent Co., Ltd. \\
\hline $\mathrm{CuSO}_{4} \cdot 5 \mathrm{H}_{2} \mathrm{O}$ & $\geq 99.0 \%$ & $7758-99-8$ & Sinopharm Chemical Reagent Co., Ltd. \\
\hline Kerosene & $1 \mathrm{~L}$ & $8008-20-6$ & Sinopharm Chemical Reagent Co., Ltd. \\
\hline Diquat dibromide & $100 \mu \mathrm{g} / \mathrm{mL}$ & $85-00-7$ & Sinopharm Chemical Reagent Co., Ltd. \\
\hline$\beta$-Humulene & $\geq 90 \%$ & $116-04-1$ & Sinopharm Chemical Reagent Co., Ltd. \\
\hline
\end{tabular}

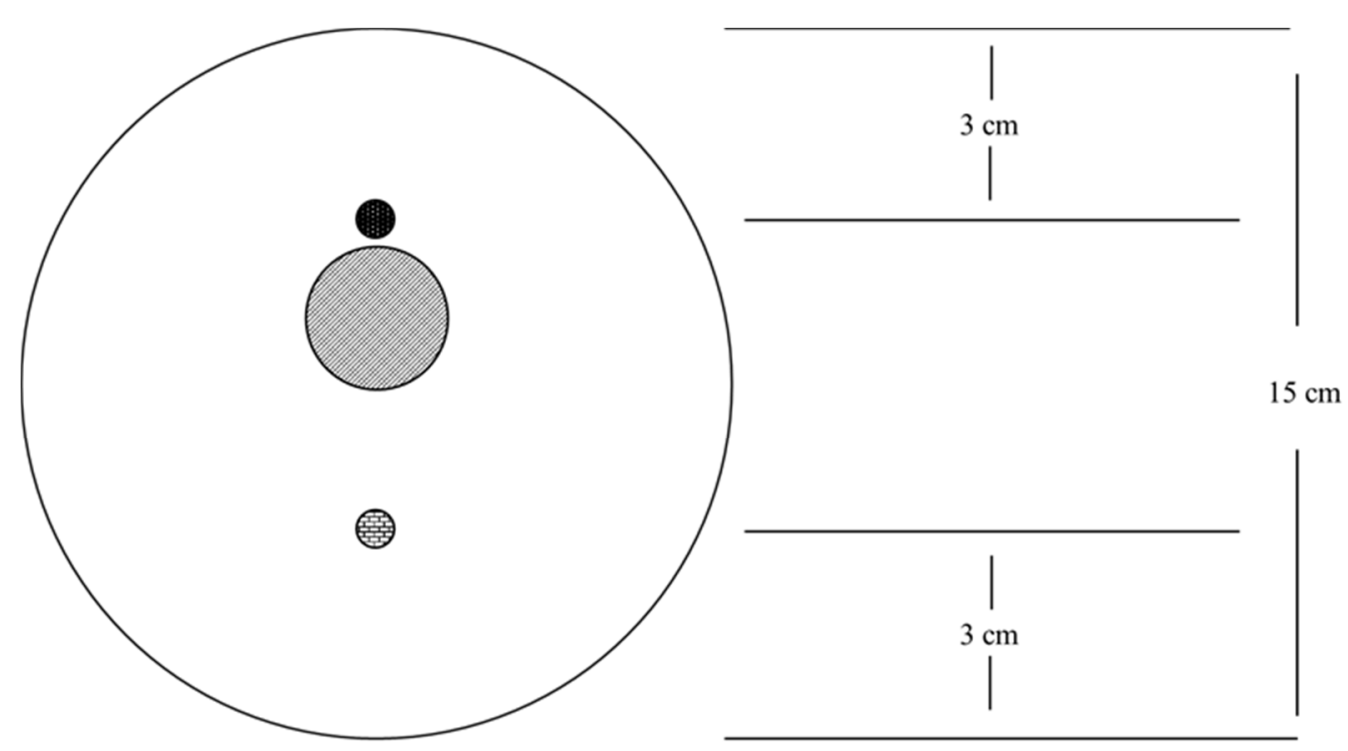

chemical substances $\bigcirc$ sampling point

Bursaphelenchus xylophilus

Figure 1. Schematic diagram of the plate attraction experiment.

These plates were then sealed with parafilm and placed in an incubator at $25^{\circ} \mathrm{C}$ for $24 \mathrm{~h}$. Agar blocks with a diameter of $3 \mathrm{~cm}$ were excised from the position of the cotton ball to which chemicals were added. PWNs in the agar blocks were separated via the Baermann funnel technique. The isolated PWNs were observed and counted under an optical microscope. 


\subsection{Attractive Effects of Several Chemicals on PWN in Infected Wood}

After P. thunbergii Parl. trees that had died in the previous year were felled in the Yaolingkou area of Jurong Forest Farm in Jiangsu Province, we removed the side branches and cut the infected wood on the main trunk into segments with a length of $60 \mathrm{~cm}$. A total of three round holes staggered on the positive and negative sides of each wood segment with a diameter of $35 \mathrm{~mm}$ and depth of $40 \mathrm{~mm}$ were introduced, with holes No. 1 and No. 3 located at either and No. 2 in the middle. PWNs in wood chips obtained by drilling were separated from the wood chips and counted to obtain the initial value.

After pretreatment, different doses of chemicals were injected into the holes in the infected wood. Eight different chemicals and three different concentration dose gradients were set up in the experiment. The holes were filled with sawdust and rolled cotton and then sealed with parafilm.

Three weeks after the chemicals were injected, approximately $50 \mathrm{~g}$ of wood chips were collected from the holes at both ends of the wood segments, from which PWNs were separated and counted to obtain the treatment value. Five wood segments were inoculated in each treatment (generally from different trees), and sterile water treatment was used as a negative control. The rate of increase in the PWN counts was calculated with the following formula, and the result was used as a standard to measure the attraction effect of the chemicals on PWNs:

$$
\mathrm{r}=\frac{\mathrm{x}_{2}-\mathrm{x}_{1}}{\mathrm{x}_{1}} \times 100 \%
$$

where ' $\mathrm{r}$ ' represents the rate of increase in the PWN counts in infected wood after chemical treatment (\%); $x_{1}$ represents the number of PWNs per gram of sawdust (dry weight) before chemical treatment; and $\mathrm{x}_{2}$ represents the number of PWNs per gram of sawdust (dry weight) after chemical treatment.

\subsection{The Self-Designed Assay for Evaluating Repellent Effects of Chemicals toward PWN}

Water agar medium $(15 \mathrm{~cm})$ was prepared via the quadripartite agar plate method and marked appropriately (Figure 2). After the water agar medium was naturally air dried, a hole was introduced with a punch at three points located $3.75 \mathrm{~cm}$ from the center of the plate, and an aseptic cotton ball was added to the hole. Sterile water was used as the negative control. Finally, $200 \mu \mathrm{L}$ of a PWN solution (approximately 1000 PWNs) was dripped at the central point in the dried medium.

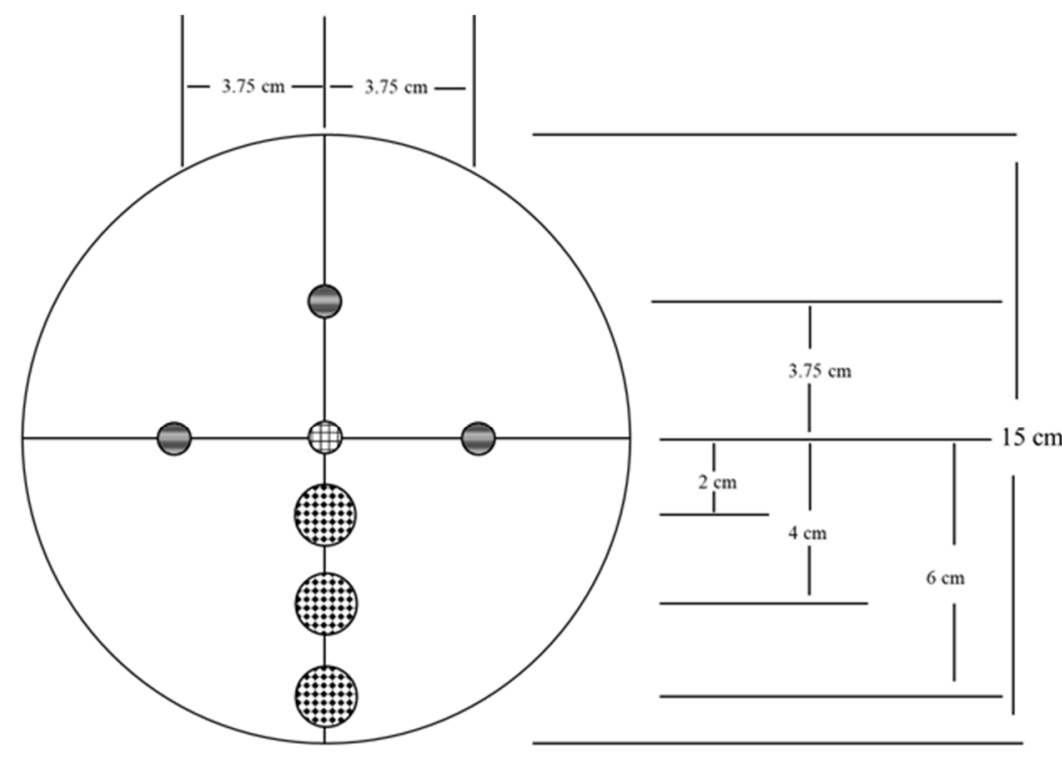

chemical substances $\bigoplus$ Bursaphelenchusxylophilus sampling point

Figure 2. Schematic diagram of the plate repellency experiment. 
After $24 \mathrm{~h}$, the movement of the PWNs was observed, agar blocks were removed within radii of $2 \mathrm{~cm}, 4 \mathrm{~cm}$ and $6 \mathrm{~cm}$ from the center of the plate, and the PWNs were separated and counted. Each process was repeated 5 times.

\subsection{Repellent Effect of Several Chemicals on PWN in Infected Wood}

After P. thunbergii Parl. that had died in the previous year were felled in the Yaolingkou area of Jurong Forest Farm in Jiangsu Province, the side branches were removed, and the main trunks of the infected wood were cut into segments with a length of $60 \mathrm{~cm}$. A total of three round holes staggered on the positive and negative sides of each wood segment with a diameter of $35 \mathrm{~mm}$ and a depth of $40 \mathrm{~mm}$ were introduce, with holes No. 1 and No. 3 located at both ends and No. 2 in the middle. PWNs in wood chips obtained by drilling were separated from the wood chips and counted to obtain the initial value.

After pretreatment, different doses of chemicals were injected into the holes in the wood that contained PWNs. Three different chemicals and three different concentration dose gradients were set up in the experiment. The holes were filled with sawdust and rolled cotton and then sealed with parafilm.

Three weeks after the chemicals we estimate the rate of decrease in the PWN as described above for the rate of the increase. The rate of the decrease in the PWN counts was calculated with the following formula, whose result was used as a standard to measure the repellent effect of the chemicals on PWN:

$$
\mathrm{r}=\frac{\mathrm{x}_{1}-\mathrm{x}_{2}}{\mathrm{x}_{1}} \times 100 \%
$$

where $r$ represents the rate of the decrease in the PWN counts on infected wood after chemical treatment (\%); $x_{1}$ represents the number of PWNs per gram of sawdust (dry weight) before chemical treatment; and $x_{2}$ represents the number of PWNs per gram of sawdust (dry weight) after chemical treatment.

\subsection{Statistical Analysis}

The data were subjected to analysis of variance and Duncan's multiple comparison test with SPSS 21.0 software (IBM Inc., Armonk, NY, United States), and the standard errors of all mean values were calculated $(p<0.05)$. Graphs were generated using GraphPad Prism 8.0 (GraphPad Software, Inc., Chicago, IL, USA).

Two analyses were carried out on the data obtained in the attractive effects experiment. Firstly, the effects of a chemical to different strains of PWNs were compared. If there was no difference of effect among the strains, the average value of the effect from the three strains was used in downstream analysis. Secondly, the effects of different chemicals to the same PWNs strain were compared.

In addition, the number of nematodes along the distance gradient was reported to detect the repellent effect of chemicals on PWNs.

Detailed analysis was described in the "supplementary file" (Table S1).

\section{Results}

\subsection{Analysis of the Attractive Effects of Several Chemicals toward PWN}

The PWNs from the cotton balls were isolated after $24 \mathrm{~h}$.

The number of PWNs in the control (sterile water treatment) was $70 \pm 0.81$, while the number of PWNs in the treatment of diacetyl, linoleic acid, oleic acid, toluene and $\beta$-myrcene, $\beta$-caryophyllene, stearic acid and palmitic acid were $515 \pm 5.89,444 \pm 4.49$, $338 \pm 5.44,291 \pm 5.73,264 \pm 8.18,168 \pm 3.68,154 \pm 3.68,148 \pm 2.45$, respectively (Table 2). The number of PWNs in the chemical treatments were all significantly higher than that in the control $(p<0.05)$, suggesting that the chemicals tested can attracted PWNs on the water agar medium. 
Table 2. Attractive effects of different chemicals toward different PWN strains.

\begin{tabular}{ccccc}
\hline PWN & AmA3 & AA3 & Yw4 & $\begin{array}{c}\text { Average } \\
\text { Data }\end{array}$ \\
\hline Chemicals & $70 \pm 5.60 \mathrm{~h} \mathrm{~A}$ & $71 \pm 14.93 \mathrm{~g} \mathrm{~A}$ & $69 \pm 2.08 \mathrm{~g} \mathrm{~A}$ & $70 \pm 0.81 \mathrm{~g}$ \\
Control & $89 \pm 5.29 \mathrm{gh} \mathrm{A}$ & $85 \pm 6.06 \mathrm{fg} \mathrm{A}$ & $76 \pm 2.49 \mathrm{fg} \mathrm{A}$ & $83 \pm 5.43 \mathrm{fg}$ \\
$\mathrm{NaClO}_{2} \mathrm{HCL}_{3} \mathrm{O}_{2}$ & $96 \pm 5.60 \mathrm{gh} \mathrm{A}$ & $97 \pm 14.93 \mathrm{fg} \mathrm{A}$ & $87 \pm 2.08 \mathrm{fg} \mathrm{AB}$ & $93 \pm 4.49 \mathrm{fg}$ \\
Clycerol & $113 \pm 2.06 \mathrm{fg} \mathrm{A}$ & $89 \pm 5.60 \mathrm{fg} \mathrm{B}$ & $96 \pm 6.33 \mathrm{fg} \mathrm{B}$ & $98 \pm 10.07 \mathrm{fg}$ \\
Lactic acid & $121 \pm 8.62 \mathrm{fg} \mathrm{A}$ & $116 \pm 3.56 \mathrm{fg} \mathrm{AB}$ & $124 \pm 10.39 \mathrm{fg} \mathrm{A}$ & $120 \pm 3.29 \mathrm{fg}$ \\
Palmitic acid & $151 \pm 8.62 \mathrm{e} \mathrm{A}$ & $148 \pm 3.56 \mathrm{ef} \mathrm{A}$ & $145 \pm 8.36 \mathrm{ef} \mathrm{A}$ & $148 \pm 2.45 \mathrm{ef}$ \\
Stearic acid & $154 \pm 11.49 \mathrm{fg} \mathrm{A}$ & $150 \pm 3.39 \mathrm{ef} \mathrm{A}$ & $159 \pm 6.06 \mathrm{ef} \mathrm{A}$ & $154 \pm 3.68 \mathrm{ef}$ \\
$\beta$-Caryophyllene & $173 \pm 3.39 \mathrm{ef} \mathrm{A}$ & $164 \pm 10.43 \mathrm{ef} \mathrm{B}$ & $169 \pm 6.06 \mathrm{ef} \mathrm{A}$ & $168 \pm 3.68 \mathrm{ef}$ \\
$\beta$-Myrcene & $272 \pm 2.49 \mathrm{~cd} \mathrm{~A}$ & $253 \pm 10.43 \mathrm{de} \mathrm{B}$ & $268 \pm 10.37 \mathrm{de} \mathrm{A}$ & $264 \pm 8.18 \mathrm{~cd}$ \\
Toluene & $292 \pm 6.06 \mathrm{~cd} \mathrm{~A}$ & $284 \pm 6.33 \mathrm{~cd} \mathrm{~A}$ & $298 \pm 2.08 \mathrm{~cd} \mathrm{~A}$ & $291 \pm 5.73 \mathrm{~cd}$ \\
Oleic acid & $337 \pm 11.49 \mathrm{c} \mathrm{A}$ & $346 \pm 2.83 \mathrm{bc} \mathrm{AB}$ & $333 \pm 5.06 \mathrm{bc} \mathrm{A}$ & $338 \pm 5.44 \mathrm{bc}$ \\
Linoleic acid & $441 \pm 4.22 \mathrm{~b} \mathrm{~A}$ & $451 \pm 3.74 \mathrm{~b} \mathrm{AB}$ & $442 \pm 3.85 \mathrm{~b} \mathrm{~A}$ & $444 \pm 4.49 \mathrm{~b}$ \\
Diacetyl & $513 \pm 8.72 \mathrm{a} \mathrm{AB}$ & $523 \pm 2.08 \mathrm{a} \mathrm{AB}$ & $509 \pm 11.4 \mathrm{a} \mathrm{A}$ & $515 \pm 5.89 \mathrm{a}$ \\
\hline
\end{tabular}

Different letters indicate significant differences at $p<0.05$. Capital letters indicate the differences among PWN strains. Lowercase letters indicate the differences among chemicals. AmA3 AA3 Yw4 means three strains of PWNs.

Chemicals that might potentially attract PWNs were screened on water agar medium and inoculated onto segments of infected wood. The PWNs present in infected wood were sampled, separated and counted (Figure 3). The increase in the PWN populations around the inoculation site differed from the infected wood which treated with different chemicals (Figure 3). The inoculation of linoleic acid, oleic acid, diacetyl and $\beta$-myrcene maximized the number of PWNs around the inoculation site, with increase rates of $54.23 \%, 49.78 \%$, $36.28 \%$ and $26.39 \%$, respectively ( $4.57 \%$ increase in the control). Different doses of the same chemical resulted in little difference in attraction effect.

It could be concluded that linoleic acid, oleic acid, diacetyl and $\beta$-myrcene can attract PWNs in infected wood. However, $\beta$-caryophyllene, palmitic acid, toluene and stearic acid cannot spread and volatilize in infected wood, so their attraction effect on PWNs is limited.

\subsection{Analysis of Repellent Effects of Several Chemicals on PWN}

The chemicals were placed at three different points on water agar medium, and $200 \mu \mathrm{L}$ of a PWN suspension (approximately 1000 PWNs) was added to the central drop. After $24 \mathrm{~h}$, the PWNs in the agar blocks with diameters of $2 \mathrm{~cm}, 4 \mathrm{~cm}$ and $6 \mathrm{~cm}$ from the central point were separated and counted. After $24 \mathrm{~h}$ of treatment with five kinds of inorganic compounds, the numbers of PWNs separated from water agar medium blocks ranged from 20 44 PWNs; in particular, the numbers of PWNs isolated from agar blocks with diameters of $2 \mathrm{~cm}$ from the center of plates treated with $\beta$-humulene, kerosene and diquat dibromide ranged from 97 134 (23 33 for the control), and the number of PWNs increased along with the diameters (Figure 4). In conclusion, $\beta$-humulene, kerosene and diquat dibromide have a repellent effect on PWNs.

After the potentially PWN-repellent chemicals were screened on water agar medium, they were inoculated onto infected woods. The rate of the decrease in PWN counts around the inoculation site differed from the infected woods which treated with different chemicals (Figure 5). The number of PWNs around the inoculation site reduced significantly by inoculation with $\beta$-humulene and kerosene, and the decrease rates reached $36.58 \%, 39.62 \%$ and $34.34 \%$, respectively ( $6.31 \%$ lower than that of the control). Therefore, the blocks of agar treated with $\beta$-humulene and kerosene can deter PWNs in infected wood. 


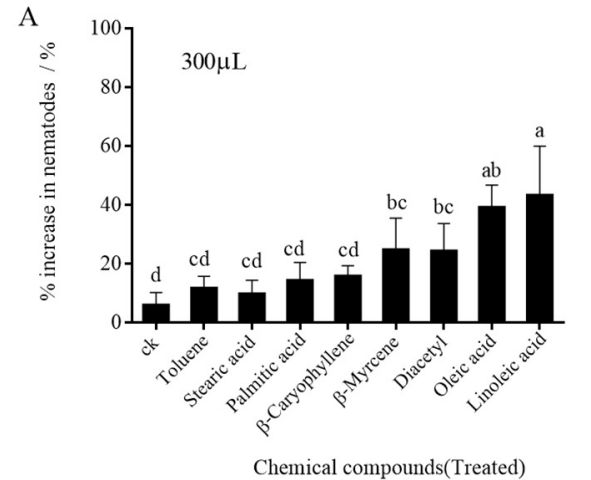

C

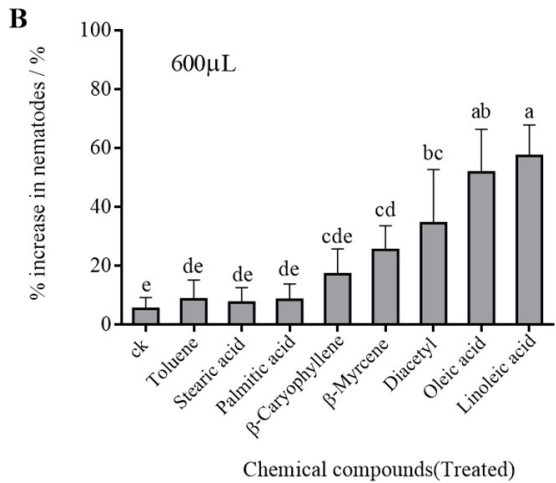

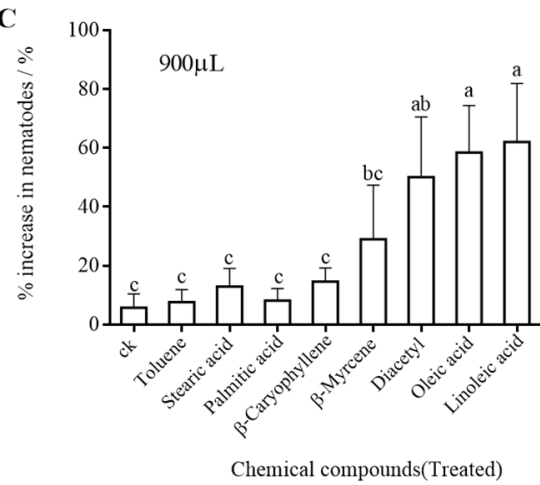

Figure 3. Changes in PWNs in infected woods treated with different attractant chemicals. (A) The dose of chemicals used was $300 \mu \mathrm{L}$; (B) The dose of chemicals used was $600 \mu \mathrm{L}$; (C)The dose of chemicals used was $900 \mu \mathrm{L}$. Lowercase letters indicate the differences among chemicals.

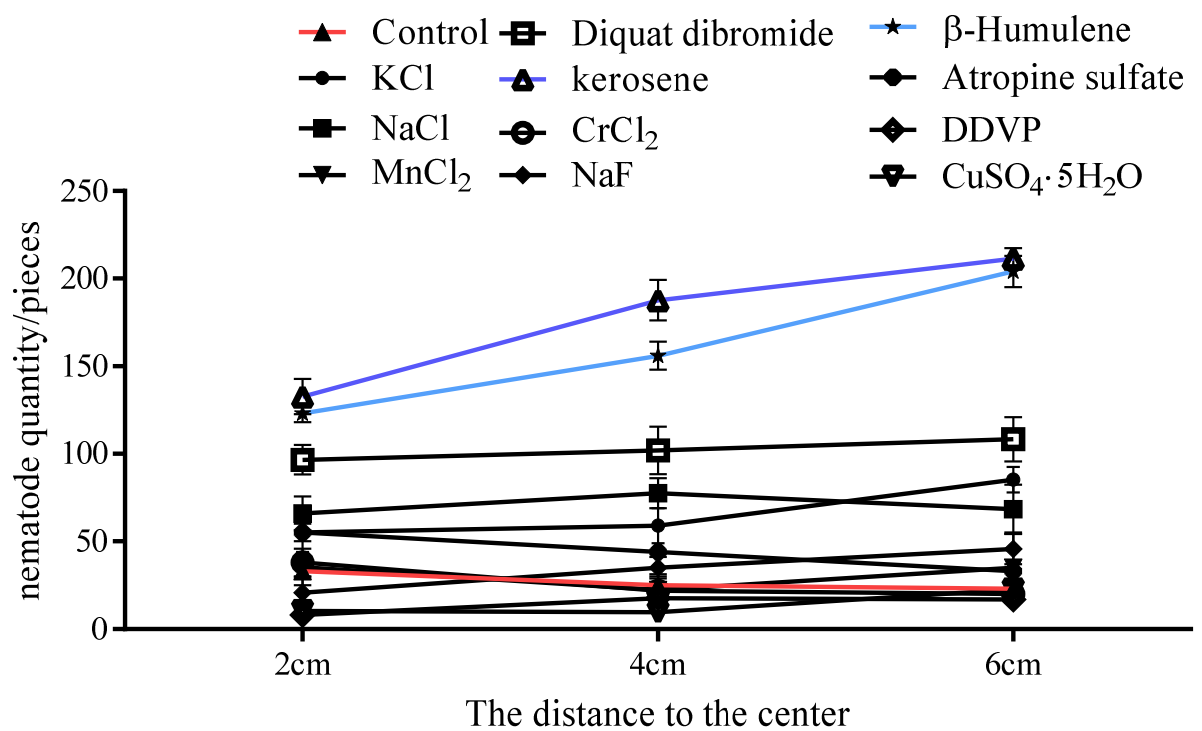

Figure 4. Movement of PWN in agar blocks after treatment with different chemicals. 


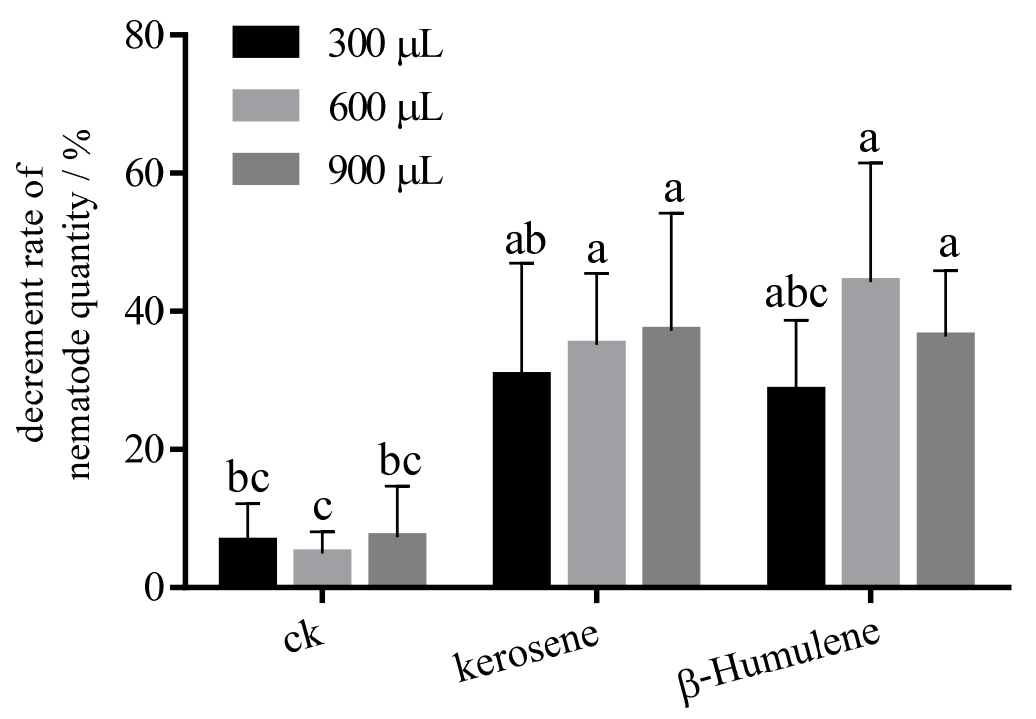

Chemical compounds(Treated)

Figure 5. Changes in the number of PWN in infected trees after treatment with different repellent chemicals. Lowercase letters indicate the differences among chemicals.

\section{Discussion}

PWD has continued to spread in China in recent years, the total occurrence areas and the number of dead trees is increasing each year, and there is an urgent need for prevention and control [15]. According to previous studies, the control and prevention strategy refer to quarantine and epidemic monitoring [16], epidemic wood removal [17] and vector insect control [18]. However, there are some limitations of current methods, such as the difficulty and high cost of treatment [19]. There are also some problems in wood disposal, such as the easy spread of the PWNs and the difficulty of monitoring [20,21]. As a result, it is imperative to block the migration of PWN with the insect vector.

The mechanism of PWN migration toward vector insects in the infection cycle of PWNs has gradually become a research focus of scholars. If chemicals with a significant effect on PWNs can be found, they can be used to interfere with their movement in infected wood, block their accumulation in the pupal chamber of M. alternatus, and effectively reduce the dispersal efficiency of PWD.

In our study, $\mathrm{NaClO}, \mathrm{C}_{2} \mathrm{HCL}_{3} \mathrm{O}_{2}$, Clycerol, and lactic acid were only able to stimulate the migration behavior of PWN, but without, chemotaxis to PWN. Palmitic acid, stearic acid, $\beta$-Caryophyllene, toluene can attract PWN in the water agar medium, However, they did not show attractive effect in the infected wood. Oleic acid, linoleic acid, diacetyl, $\beta$-Myrcene can attract PWN, and were able to stably permeate and volatilize within pine trees. On the other hand, only kerosene and $\beta$-humulene had repellent effects on PWN among 11 chemicals tested.

The tropic effects of chemicals on PWNs have been reported in previous studies. Abietic acid and phytol were found to exist in pine resin and exert a strong attractive effect on PWN, which may be related to the infection of pine trees by PWN [16]. $\beta$ Caryophyllene, $\beta$-phellandrene and isolongifolene were proven to be able to attract PWNs in assays involving the inoculation of water agar or infected wood [22,23]. Streptomyces plicatus were proved to be have attractive ability to Meloidogyne incognita at $72 \mathrm{~h}$ and negative to the Caenorhabditis elegans at $24 \mathrm{~h}$ [14]. Besides, rhizosphere bacteria are able to produce volatile substances, as signal factors to attract or repel nematodes [24,25]. In this experiment, we verified the attractivity of several chemicals on water agar medium and infected wood, which were tested similarly by Tan and Du [26,27]. Compared with the similar experiment, the method we used here was improved, and other attractant substances besides terpenes were found. 
At present, some scholars have studied the repellent effect on the Caenorhabditis elegans, but there are few similar studies on PWN, researches on the chemotaxis of PWNs are focused mainly on the attractive effects of chemicals on PWNs. While this study proved that chemicals exerted a repellent effect on PWN in the infected wood, and the repellent effect was enhanced with an increasing volume.

Li pointed out that the chemotaxis of Caenorhabditis elegans toward different concentrations of diacetyl were inhibited by metal ions [5]. However, this situation was not in our study, and the metal ions did not have repellent effect on PWN. On the other hand, in this study mixture of pesticide with strong smell have repellent effect on PWN yet.

In this study, a total of 7 chemicals screened from the aspects of attraction and repellency were shown to significantly affect the movement of PWNs in infected wood to interfere with the normal pupal chamber effect. However, after the infected wood is cut down, the internal resin flow stops, and the effects of chemicals then depend on their own diffusivity and volatility, so there are some limitations of this approach. However, by inhibiting the pupal chamber effect, which is a key link in the infection cycle, the transmission of PWNs to other healthy pine trees by their vector insects to complete the infection cycle is prevented. Hence, the spread of the disease can be alleviated to a certain extent.

\section{Conclusions}

This study explored the effects of several chemicals on the movement behavior of PWNs from the aspects of attraction and repellency. It indicates that diacetyl, linoleic acid, oleic acid, toluene, $\beta$-myrcene, $\beta$-caryophyllene, stearic acid and palmitic acid could attract PWNs, and $\beta$-humulene kerosene could repel PWNs on the wood newly killed by PWNs. The use of chemicals to treat infected wood for the control of PWD is technically feasible and presents broad application prospects, making it worthy of further study.

Supplementary Materials: The following are available online at https://www.mdpi.com/article/ 10.3390/f12060771/s1, Table S1. Analysis of PWN in agar blocks after treatment with different chemicals.

Author Contributions: X.-F.S.: designed the study, conducted the experiment, performed the data analysis and wrote the article; X.-L.D.: guided the article writing and data analysis; Q.-T.W. \& L.-N.S.: collected the samples and performed data analysis; J.-R.Y.: guarantor of the integrity of the entire study and approved the final version of the manuscript. All authors have read and agreed to the published version of the manuscript.

Funding: This research was funded by the Major Emergency Science and Technology Project of National Forestry and Grassland Administration, PRC (Grant Number: ZD202001).

Informed Consent Statement: Informed consent was obtained from all subjects involved in the study.

Data Availability Statement: The data presented in this study are available on request from the corresponding author.

Conflicts of Interest: The authors declare no conflict of interest.

\section{References}

1. Zhao, L.; Zhang, X.; Wei, Y.; Zhou, J.; Zhang, W.; Qin, P.; Chinta, S.; Kong, X.; Liu, Y.; Yu, H. Ascarosides coordinate the dispersal of a plant-parasitic nematode with the metamorphosis of its vector beetle. Nat. Commun. 2016, 7, 12341. [CrossRef] [PubMed]

2. Song, Y.; Zang, X.; Liu, Y.; Wang, X. A preliminary study on population dynamics of Bursaphelenchus xylophilus in diseased trees. For. Pest Dis. 1993, 15, 9-11.

3. Jia, W.; Kong, X.; Wang, X.; Ren, L.; Zhu, X. In A rapid $\beta$-Myrcene-attractant assisted wood sampling method for PCR-based detection of Bursaphelenchus xylophilus in Pinus massoniana wood tissue. In Proceedings of the 2011 International Conference on Remote Sensing, Environment and Transportation Engineering, Nanjing, China, 24-26 June 2011.

4. Zhang, K.; Liang, J.; Yan, D.; Zhang, X. Research Advances of Pine Wood Nematode Disease in China. World For. Res. 2010, 23, 59-63.

5. Li, M.; Li, H.; Sheng, R.-C.; Sun, H.; Sun, S.-H.; Chen, F.-M. The First Record of Monochamus saltuarius (Coleoptera; Cerambycidae) as Vector of Bursaphelenchus xylophilus and Its New Potential Hosts in China. Insects 2020, 11, 636. [CrossRef] 
6. Zhao, L.; Mota, M.; Vieira, P.; Butcher, R.A.; Sun, J. Interspecific communication between pinewood nematode, its insect vector, and associated microbes. Trends Parasitol. 2014, 30, 299-308. [CrossRef] [PubMed]

7. Zhao, L.; Zhang, S.; Wei, W.; Hao, H.; Zhang, B.; Butcher, R.A.; Sun, J. Chemical Signals Synchronize the Life Cycles of a Plant-Parasitic Nematode and Its Vector Beetle. Curr. Biol. CB 2013, 23, 2038-2043. [CrossRef]

8. Zhang, X.; Feng, L.; Chinta, S.; Singh, P.; Wang, Y.; Nunnery, J.K.; Butcher, R.A. Acyl-CoA oxidase complexes control the chemical message produced by Caenorhabditis elegans. Pro. Natl. Acad. Sci. USA 2015, 112, 3955-3960. [CrossRef] [PubMed]

9. Chen, X.; Xu, R.; Xie, B. The role of chemical communication in the infection and spread of pine wood nematodes (Bursaphelenchus xylophilus). Acta Ecol. Sin. 2005, 22, 339-345.

10. Wang, Y.; Chen, J.; Chen, F. Transmission of Bursaphelenchus xylophilus (Nematoda: Aphelenchoididae) through feeding activity of Monochamus alternatus (Coleoptera: Cerambycidae). J. Nanjing For. Univ. 2019, 43, 1-10.

11. Ding, X.L. The Pathogenic Mechanism and Virulence Variations Analyses of Bursaphelenchus xylophilus Based on high-Throughput Sequencing. Ph.D. Thesis, Nanjing Forestry University, Nanjing, China, 2016.

12. Zeng, Y.X.; Kong, Q.; Li, Y.; Jing, Y.M. Effects of chemicals with different toxicity levels on the perceptual behavior of nematodes. J. Toxicol. 2014, 28, 47-53.

13. Wicks, S.R.; Vries, C.J.D.; Luenen, H.G.A.M.; Plasterk, R.H.A. CHE-3, a cytosolic dynein heavy chain, is required for sensory cilia structure and function in Caenorhabditis elegans. Dev. Biol. 2000, 221, 295-307. [CrossRef]

14. Yang, L.L. Chemotaxis of Two Kinds of Nematodes Induced by Rhizosphere Bacteria. Master Thesis, Yunnan University, Kunming, China, 2016.

15. Ye, J.R. Epidemic Status of Pine Wilt Disease in China and Its Prevention and Control Techniques and Counter. Sci. Silvae Sin. 2019, 55, 1-10.

16. Liu, K.C.; Zeng, F.L.; Ben, A.L.; Han, Z.M. Pathogenicity and repulsion for toxin-producing bacteria of dominant bacteria on the surface of American Pine Wood Nematodes. Phytopathology 2017, 165, 580-588. [CrossRef]

17. Wang, X.Z.; Cao, Y.F.; Wang, L.F.; Piao, C.G.; Li, C.L. Current status of pine wilt disease and its control status. J. Environ. Entomol. 2018, 40, 256-267.

18. Zheng, L.P. Technology for in situ control of pine wilt nematode disease in forest. Modern. Agric. Sci. Technol. $2017,2,106-107$.

19. FUTAI, K. Population Dynamics of Bursaphelenchus lignicolus (Nematoda: Aphelenchoididae) and B. mucronatus in Pine Seedlings. Appl. Entomol. Zool. 1980, 15, 458-464. [CrossRef]

20. Zhu, L.H.; Ye, J.R.; Negi, S.; Xu, X.L.; Wang, Z.L.; Ji, J.Y. Pathogenicity of Aseptic Bursaphelenchus xylophilus. PLoS ONE 2012, 7, e38095.

21. Zhang, B.; Zhang, W.; Lu, M.; Ahmad, F.; Tian, H.; Ning, J.; Liu, X.; Zhao, L.; Sun, J. Chemical Signals of Vector Beetle Facilitate the Prevalence of a Native Fungus and the Invasive Pinewood Nematode. J. Nematol. 2017, 49, 341.

22. Shuto, Y.; Watanabe, H. Attractants from a vector, Monochamus alternatus, for the pine wood nematode. J. Agric. Chem. Soc. Jpn. 1987, 51, 1457-1458.

23. Bolla, J.A. Attraction of Bursaphelenchus xylophilus, Pathotype MPSy-1, to Monochamus carolinensis Larvae. Jpn. J. Nematol. 1989, 32-37. [CrossRef]

24. Pan, J.L.; Yao, H.W. Analysis of the epidemic situation of pine wilt disease in China in 2019. For. Pest Dis. 2020, $40,32-37$.

25. Sun, Y. Effect of Rhizosphere Microbe on Chemotaxis of Root-Knot Nematode. Master's Thesis, Yunnan University, Kunming, China, 2017.

26. Du, J. Analysis effects of Several Pine Volatiles on Behavior of Bursaphelenchus xylophilus. Farmers Consult. 2020, 98, 98-101.

27. Tan, J.J.; Hao, D.J. Effects of Several Pine Volatiles on Behavior of Bursaphelenchus xylophilus. J. Northeast For. Univ. 2009, 12, 58-59. 